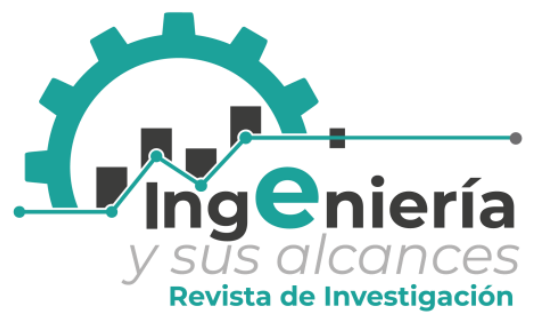

Ingeniería y sus alcances, Revista de Investigación Https://doi.org/10.33996/revistaingenieria.v4i10.68 Septiembre - diciembre 2020 Volumen 4 / No. 10 ISSN: $2664-8245$ ISSN-L: $2664-8245$ pp. $233-252$

Revista de Investigación

\title{
Sistema automatizado para regular el comportamiento de los factores climáticos en el cultivo de hortalizas
}

\author{
Automated system to regulate the behavior of climatic factors in the \\ cultivation of vegetables
}

\author{
Sistema automatizado para regular o comportamento dos fatores climáticos \\ no cultivo de hortaliças
}

\section{Mónica Joselin Ala Delgado \\ kinmo.ad127@gmail.com \\ ORCID 0000-0001-5784-5520 \\ Universidad Privada Domingo Savio, Bolivia}

Artículo recibido mayo 2020 | Arbitrado en julio 2020 | Publicado en septiembre 2020

\section{RESUMEN}

La presente investigación tuvo como objetivo proponer un sistema automatizado para regular el comportamiento de los factores climáticos en el cultivo de hortalizas en el invernadero solar la lechuguita de la ciudad de Potosí. La metodología que se usó para el desarrollo de la investigación fue descriptiva-propositivo, bajo un enfoque mixto, con un diseño documental y de campo. Los métodos que se usaron en la investigación fueron, histórico-lógico, análisis-síntesis, otros. Las técnicas e instrumentos utilizados para la recolección de datos fueron la observación (guía), encuesta (cuestionario) y entrevista (guía). La población y muestra, estuvo conformada por 16 agricultores de invernaderos solares del sector villa Concepción y la Lechuguita. Se obtuvo como resultado, que no cuentan con los factores climáticos adecuados, generando que los cultivos puedan ser afectados. Se comprobó que los agricultores con el sistema automatizado controlan todos los factores y realizan monitoreo en tiempo real.

Palabras clave: Invernaderos; modelos de invernaderos solares; factores climáticos, metodología XP

\section{ABSTRACT}

RESUMO
The objective of this research was to propose an automated system to regulate the behavior of climatic factors in the cultivation of vegetables in the solar greenhouse, the lechuguita of the city of Potosí. The methodology used for the development of the research was descriptive-propositional, under a mixed approach, with a documentary and field design. The methods used in the research were historical-logical, analysissynthesis, others. The techniques and instruments used for data collection were observation (guide), survey (questionnaire) and interview (guide). The population and sample consisted of 16 farmers from solar greenhouses in the Villa Concepción and La lechuguita sectors. It was obtained as a result, that they do not have the appropriate climatic factors, causing the crops to be affected. Farmers with the automated system were found to control all factors and perform real-time monitoring.

Key words: Greenhouses; models of solar greenhouses; climatic factors, XP methodology
O objetivo desta pesquisa foi propor um sistema automatizado para regular o comportamento dos fatores climáticos no cultivo de hortaliças em estufa solar, a lechuguita da cidade de Potosí. A metodologia utilizada para o desenvolvimento da pesquisa foi descritivo-propositivo, sob abordagem mista, com desenho documental e de campo. Os métodos utilizados na pesquisa foram histórico-lógico, análise-síntese, outros. As técnicas e instrumentos utilizados para a coleta de dados foram observação (guia), levantamento (questionário) e entrevista (guia). A população e a amostra consistiram de 16 agricultores de estufas solares nos setores de Villa Concepción e La Lechuguita. Obteve-se como resultado, que não possuem os fatores climáticos adequados, fazendo com que as lavouras sejam afetadas. Os agricultores com o sistema automatizado conseguiram controlar todos os fatores e realizar o monitoramento em tempo real.

Palavras chave: Greenhouses; modelos de estufas solares; fatores climáticos, metodologia XP 


\section{INTRODUCCIÓN}

El proceso agropecuario, es uno de los pilares de la economía donde tiene el propósito de cultivar la tierra, mediante diferentes alternativas y tratamientos pero con el fin de obtener frutos o vegetales, es por eso que se practicó la agricultura urbana que es más conocido como producción a campo abierto, donde fue una tendencia desde años remotos, se puso en práctica para combatir con la pobreza y generar empleos, pero como desventaja se tuvo a los fenómenos climatológicos, puesto que esto fue uno de los grandes problemas porque no se podía tener el cuidado del cultivo y esto generaba pérdidas en la producción.

Las pérdidas de productos hicieron que algunos de los agricultores migraran a los cultivos bajo invernaderos solares, para tener el cuidado de su producción. Cabe mencionar que un invernadero solar es un espacio cerrado, donde mantiene su clima apropiado, además protegen a los cultivos de heladas, granizos, vientos y entre otros factores climatológicos cuyos cambios se manifiestan a distinta escala, que puedan afectar a la producción, además permite tener el control del ambiente interno de los factores climáticos.

La característica principal de este tipo de cultivo bajo invernadero solar es de tomar en cuenta los factores climáticos de cada cultivo, se entiende por factores climáticos o parámetros a la humedad, temperatura y dióxido de carbono los que influyen en el ambiente interno del invernadero, ya que depende mucho del control de estos el cuidado y desarrollo del cultivo.

En la actualidad algunos países han optado por sistemas automatizados con resultados sorprendentes, que han logrado tener una producción diaria de productos y tener la menor taza de pérdida, las ventajas son múltiples al reemplazar de lo manual a lo automático, donde la mano del hombre solo es necesaria para la siembra, esta tecnología ofrece beneficios al agricultor brindando un control adecuado, los cuales ayudan a tener calidad y cuidado en la producción del cultivo.

Esta tecnología de sistemas automatizados está entrando a Bolivia, donde ya fue inaugurado en Sipe Cochabamba. El centro de cooperación Coreana Kopia, en coordinación con el Instituto Nacional de Innovación Agropecuaria y Forestal (Iniaf), fueron los que implementaron este invernadero inteligente, que permite tener el control de las condiciones de la temperatura interna. Según el investigador de Kopia Richard Delgadillo, menciona que esta tecnología es poco común en Bolivia, en Santa Cruz existen dos invernaderos con características algo similares. Sin embargo, Potosí aún no cuenta con esta tecnología de automatización de invernaderos solares, todos los procesos son de manera tradicional.

En el invernadero solar la lechuguita, se realizó una entrevista al propietario del lugar, donde menciona que en muchas ocasiones descuida los cultivos por no contar con tiempo ni personal suficiente para hacerse responsable para el monitoreo y control del mismo, por lo cual le genera problemas de pérdidas en la producción.

En este sentido, la investigación tiene como objeto de estudio la agrónica. Siendo el campo de acción: regular el comportamiento de los factores climáticos en el cultivo de hortalizas en el invernadero solar la Lechuguita de la ciudad de Potosí.

Por lo tanto, en correspondencia con el objeto de estudio y el campo de acción, para resolver el problema de la investigación 
tiene como objetivo, proponer un sistema automatizado para regular el comportamiento de los factores climáticos en el cultivo de hortalizas en el invernadero solar la lechuguita de la ciudad de Potosí.

De esta manera con el presente estudio, se irá integrando las tecnologías de electrónica basadas en una plataforma de hardware libre sobre las cuales se realizará en un lenguaje compatible, mediante el cual se pueda monitorear los factor climáticos de un invernadero solar para el cultivo de hortalizas desde la aplicación vía bluetooth.

\section{Marco teórico}

\section{Invernaderos}

Según Juárez (2011), un invernadero es:

Una construcción agrícola con una cubierta traslúcida que tiene por objetivo reproducir o simular condiciones climáticas adecuadas para el crecimiento y desarrollo de plantas de cultivo establecidas en su interior, con cierta independencia del medio exterior. De las estructuras empleadas para proteger cultivos, los invernaderos permiten modificar $\mathrm{y}$ controlar de forma más eficiente los principales factores ambientales que intervienen en el crecimiento $\mathrm{y}$ desarrollo de las especies vegetales. (p. 2).

De acuerdo a la definición se menciona que dentro un invernadero con materiales transparentes ayuda al desarrollo y crecimiento de los cultivos, teniendo las condiciones climáticas adecuadas para cada una de las plantas, además que estos factores pueden ser controlados o manipulados de la manera más eficiente.
Estrada (2012), define invernadero como "una construcción cuya cubierta o techo es de un material que deja pasar la luz solar, facilitando la acumulación de calor durante el día y desprendiéndolo lentamente durante la noche, cuando las temperaturas descienden drásticamente", además agrega que "un invernadero permite controlar el ambiente interno, modificando el clima y creando las condiciones para el desarrollo de los cultivos en cualquier época del año"(s/p).

De acuerdo a la definición un invernadero tiene la cubierta transparente, permitiendo pasar la luz solar, donde la acumulación del sol durante el día ira afectando en la noche, y de esta manera evitar pérdidas ocasionados por la helada o cualquier otro factor que afecte al cultivo. El invernadero permite controlar el ambiente interno del invernadero modificándolo de acuerdo a las necesidades del cultivo en cualquier época del año.

\section{Modelos de invernaderos solares}

Según InfoAgro (s/f), la conformación estructural de los invernaderos se puede clasificar en:

\section{Invernadero plano o tipo parral}

Este tipo de invernadero se utiliza en zonas poco lluviosas, aunque no es aconsejable su construcción. La estructura de estos invernaderos se encuentra constituida por dos partes claramente diferenciadas, una estructura vertical y otra horizontal:

La estructura vertical está constituida por soportes rígidos que se pueden diferenciar según sean perimetrales (soportes de cerco situados en las bandas y los esquineros) o interiores (pies derechos).

La estructura horizontal, está constituida por dos mallas de alambre galvanizado 
superpuestas, implantadas manualmente de forma simultánea a la construcción del invernadero y que sirven para portar y sujetar la lámina de plástico. Los invernaderos planos tienen una altura de cubierta que varía entre 2,15 y 3,5 m y la altura de las bandas oscila entre 2 y 2,7 m. Los soportes del invernadero se apoyan en bloques tronco piramidal prefabricado de hormigón colocados sobre pequeños pozos de cimentación.

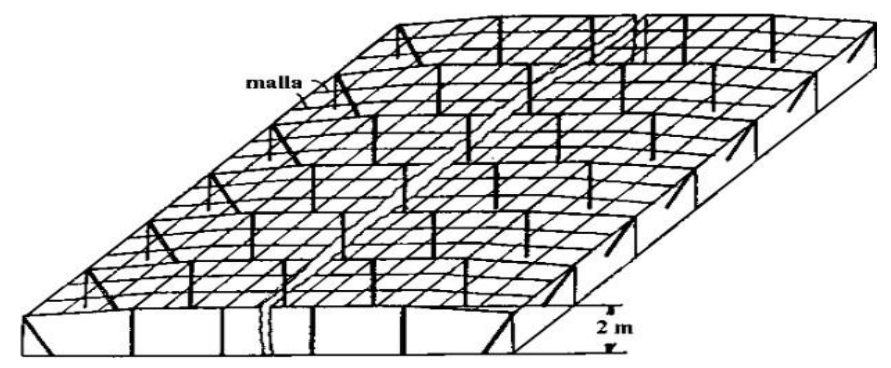

Figura 1. Modelo de invernadero utilizada en zonas poco lluviosas. Fuente: (InfoAgro, s.f.)

\section{Invernadero en raspa y amagado}

Su estructura es muy similar al tipo parral pero varía la forma de la cubierta. Se aumenta la altura máxima del invernadero en la cumbrera, que oscila entre 3 y 4,2 m, formando lo que se conoce como raspa. En la parte más baja, conocida comoamagado, se unen las mallas de la cubierta al suelo mediante vientos y horquillas de hierro que permite colocar los canalones para el desagüe de las aguaspluviales. La altura del amagado oscila de 2 a 2,8 m, la de las bandas entre 2 y $2,5 \mathrm{~m}$.

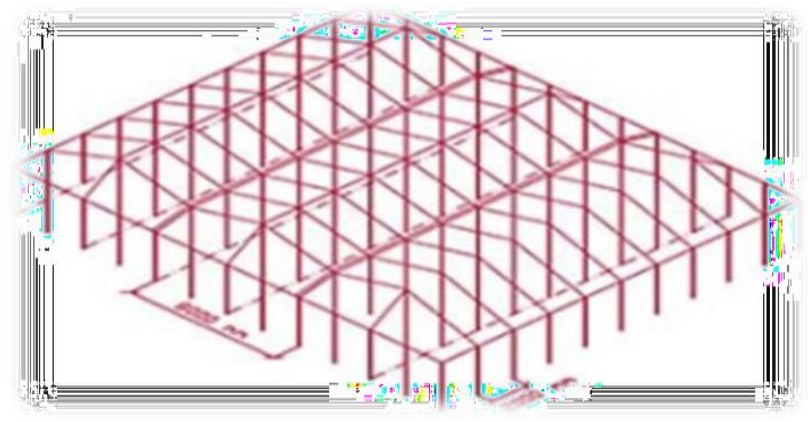

Figura 2. Modelo de invernadero su estructura es similar al tipo parral. Fuente: (InfoAgro, s.f.) 


\section{Invernadero asimétrico o inacral}

Difiere de los tipo raspa y amagado en el aumento de la superficie en la cara expuesta al sur, con objeto de aumentar su capacidad de captación de la radiación solar. Para ello el invernadero se orienta en sentido este-oeste, paralelo al recorrido aparente del sol. La inclinación de la cubierta debe ser aquella que permita que la radiación solar incida perpendicularmente sobre la cubierta al mediodía solar durante el solsticio de invierno, época en la que el sol alcanza su punto más bajo.

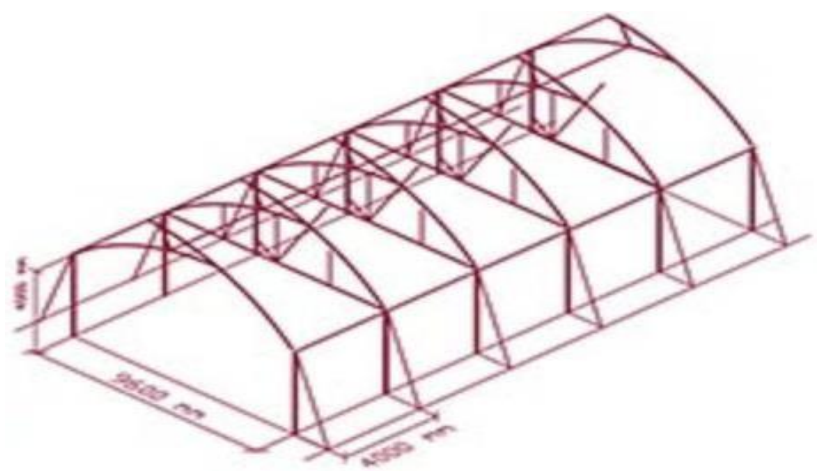

Figura 3. Modelo de invernadero asimétrico o inacral. Fuente: (InfoAgro,s.f)

Según Alvarado y Urrutia (2003), Según existen 3 tipos o formas de invernaderos:

\section{Invernadero tipo caseta}

El invernadero tipo caseta aparecieron al interior de Ovalle, en el Palqui por la década de los 60, tenían poca altura, techo plano, se les ubicaba en las laderas de los cerros preferentemente con exposición norte y todos eran de $2000 \mathrm{~m}^{2}$. Este tipo de estructura se mantuvo por muchos años, e incluso se encuentra, pero tiende a desaparecer por el problema de falta de ventilación en su sector central.

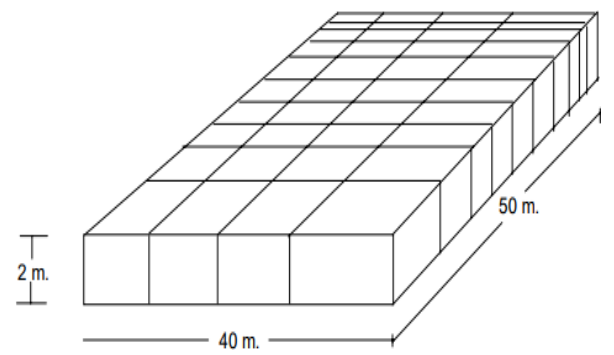

Figura 4. Modelo de invernadero usado en la década de los 60. Fuente: (Alvarado y Urrutia, 2003) 


\section{Invernadero tipo túnel}

El invernadero metálico tipo túnel, fabricado a base de tubos de acero galvanizado. Este tipo de invernadero muy fácil de instalar ha perdido interés entre los productores de tomate por su baja altura.

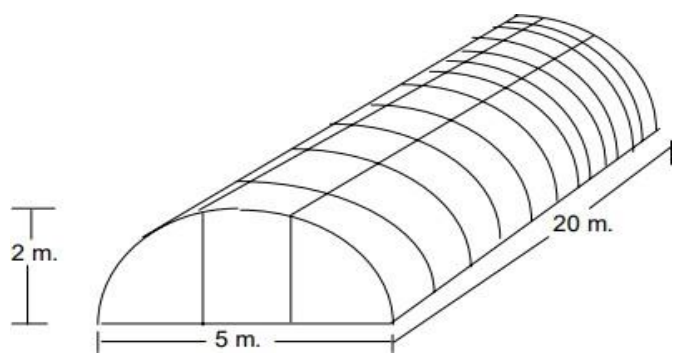

Figura 5. Modelo de invernadero usado para la producción de tomate Fuente: (Alvarado y Urrutia, 2003)

\section{Invernadero tipo capilla a dos aguas en su variante sin lucarna y con lucarna}

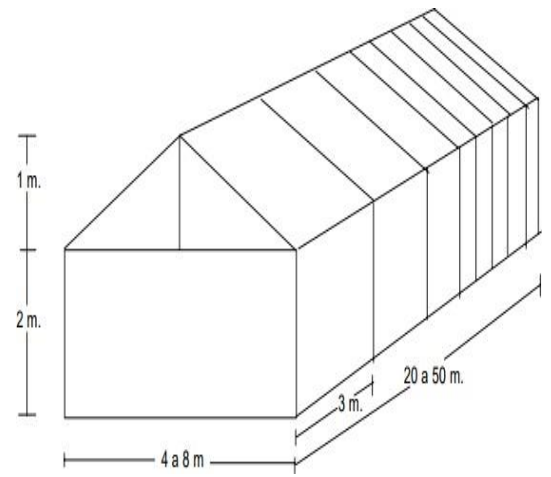

Figura 6. Modelo de invernadero tipo capilla a dos aguas también conocido variante sin lucarna. Fuente: (Alvarado y Urrutia, 2003).

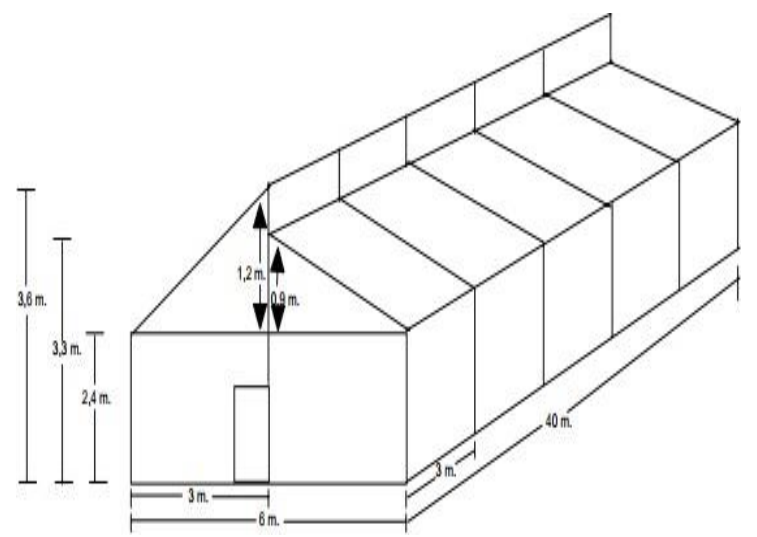

Figura 7. Modelo de invernadero tipo capilla a dos aguas también conocido variante con lucarna. Fuente: (Alvarado y Urrutia, 2003). 
El hecho de estar las naves unidas en sus costados hace que la ventilación deba ser cenital y para ello se utilizan lucarnas y aberturas frontales (anterior y posterior) para el ingreso de aire exterior.

El modelo que se utilizó en la investigación es el invernadero capilla a dos aguas también conocido variante con lucarna, porque con ella, se tiene un mejor control en las labores de producción de la manera más adecuada, las naves unidas a sus costados hacen que la ventilación sea cenital y posee un buen reparto de luminosidad en el interior.

\section{Clasificación de los Invernaderos}

Según la FAO (2004), "los invernaderos pueden ser clasificados en relación con el control de los factores meteorológicos en: climatizados, semi-climatizados y no climatizados". (pp. 19-20).

Climatizados. Son los que poseen mecanismos eléctricos, electrónicos y mecánicos de accionamiento automático para el control de temperatura, humedad relativa, ventilación y luz, usan energía transformada en sus actividades normales y su empleo depende de una explotación agrícola económicamente rentable y elevada.

Semi-climatizados. Los invernaderos semi-climatizados están dotados de cierto grado de automatización en lo relacionado a control de temperatura, humedad y luz, y se usan para explotaciones agrícolas altamente rentables.

No climatizados Los invernaderos no climatizados son, por el momento, los más viables económicamente para el pequeño y mediano productor con vistas a la producción comercial de hortalizas para el mercado nacional, no poseen ningún tipo de equipo que emplee energía transformada y su utilización está condicionada a la aplicación.

Para la investigación se tomó la clasificación de los invernaderos climatizados, ya que, se automatizara estos factores climáticos para el invernadero y tener un mejor cuidado de los cultivos.

\section{Factores climáticos}

De acuerdo a InfoAgro (s.f.), los factores climáticos o parámetros importantes a considerar dentro un invernadero son: humedad relativa, temperatura, iluminación y CO2, estos son los que influyen más en el ambiente interno del invernadero, dependerá mucho del control de estos parámetros para el desarrollo y crecimiento del cultivo de las plantas.

Por otro lado, Scuitto y Mascareña (2000), los factores climáticos a considerar son: radiación solar temperatura, humedad relativa y $\mathrm{CO} 2$, estos factores son los que influyen en un cultivo dentro de un invernadero solar, la producción y calidad del cultivo dependerá mucho de cómo se controle estos factores ya que depende mucho el rendimiento de la producción, ya que cada cultivo tiene su propio factor climático.

\section{MÉTODO}

El tipo de investigación que se utilizó para el desarrollo de la investigación estuvo centrado por el tipo descriptivo-propositivo; descriptivo, basado en la producción en invernaderos solares y los factores climáticos que intervienen en los cultivos, para conocer las situaciones importantes del cultivo de hortalizas y poder regular mejor el control de humedad, temperatura y dióxido de carbono. El diagnóstico permitió observar y describir los resultados de la situación real 
a través de los instrumentos aplicados. Y propositivo, porque se planteó una solución a la propuesta que permite regular los factores climáticos a través de un sistema automatizado, que responde a las necesidades detectadas del ambiente interno del invernadero solar y la taxonomía del cultivo.

El enfoque de análisis utilizado en la investigación fue cualitativo, ya que, permitió describir dentro del marco teórico los conceptos para un mejor entendimiento en profundidad de los factores climáticos en invernaderos solares, dentro del diagnóstico se analizó los datos obtenidos con los instrumentos utilizados. Y cuantitativo, porque permitió analizar los datos estadísticos que se recolectaron a través de los instrumentos para poder analizar e integrar datos acerca de los factores climáticos en invernaderos solares, para obtener mejores resultados dentro del diagnóstico.

Seguidamente, el diseño de la investigación que se utilizó fue documental y de campo; la investigación documental, permitió tener la accesibilidad a la búsqueda de información y el establecimiento teórico de la producción en invernaderos solares y su respectiva conceptualización. Por otra parte, la investigación de campo se utilizó para recolectar datos de los factores climáticos en invernaderos solares del cultivo de hortalizas de manera directa.

A su vez, los métodos que se usaron en la investigación fueron:

Histórico-lógico. Este método se empleó dentro del marco teórico, haciendo una revisión bibliográfica de manera detallada de la producción de hortalizas a campo abierto y bajo invernaderos solares en un proceso cronológico tanto en tiempos pasados como en la actualidad tanto en nuestro contexto como a nivel nacional y mundial. Se analizó la trayectoria concreta de la teoría en orden lógico como fue evolucionando la producción de las hortalizas bajo invernadero y los factores climáticos.

Análisis-síntesis. El primero, permitió conocer las características y cualidades de la producción de hortalizas bajo invernadero y sus factores climáticos, hasta llegar a ser analizados todos sus elementos fundamentales que la conforman. El segundo, porque se utilizó para ver la relación que existe en cada una de las partes analizadas y de qué manera influye los factores climáticos dentro un invernadero solar para la producción de las hortalizas.

Inductivo- deductivo. Inductivo, permitió analizar la información detallada, partiendo de los resultados particulares del comportamiento de los factores climáticos en cultivo de hortalizas dentro un invernadero solar, para una mejor perspectiva de los componentes que comprenden la investigación. Y deductivo, porque permitió plantear una posible solución al problema detectado abstrayendo los elementos compuestos más importantes para direccionar de mejor manera los factores climáticos en invernaderos solares.

Modelación. Se utilizó este método para modelar todos los procesos y actividades que se realizaron dentro de la producción en el invernadero solar la Lechuguita de la ciudad de Potosí, para así comprender de una manera más detallada sobre el comportamiento de los factores climáticos en hortalizas, utilizando la metodología XP y el uso de Fritzing para la estructura del circuito electrónico del sistema.

Sistémico. Permitió conocer la realidad de los invernaderos solares sobre el proceso de control de los factores climáticos, para 
luego interrelacionar todos los elementos del sistema como ser: usuario, base de datos, transacciones, procesos e informes para luego estructurarla como una sola.

Por otro lado, las técnicas e instrumentos utilizados para la recolección de datos fueron la observación, encuesta y entrevista.

Observación. Se realizó una guía de observación, para evidenciar de manera directa todos los procesos que realiza el invernadero solar la Lechuguita en la producción de hortalizas y en los invernaderos solares del sector villa Concepción, que tiene el fin de conocer que controles se realizan de los factores climáticos, de esa manera conocer la realidad y obtener datos confiables.

Encuesta. Se aplicó un cuestionario a los dueños de los siete invernaderos de la zona villa concepción de la ciudad de Potosí para recabar información acerca del comportamiento de la humedad, temperatura y dióxido de carbono (CO2), y si cuentan con las herramientas necesarias para el control del cultivo de hortalizas a través del instrumento.

Entrevista. Se aplicó la guía de entrevista a los dueños del invernadero solar la Lechuguita de la ciudad de Potosí para recabar información sobre como maneja el control de los factores climáticos en el cultivo de hortalizas, la entrevista empleada fue abierta, para indagar cual es el estado actual del cultivo en el invernadero.
La población y la muestra utilizada para la recolección de datos, estuvo conformada por los agricultores de distintos invernaderos solares $y$ dueños del invernadero la lechuguita del sector villa concepción de la ciudad de Potosí, haciendo un total de 16 personas.

Como la población utilizada en la investigación fue muy pequeño, la muestra fue censal por la cantidad de personas, "si la población es menor a cincuenta (50) individuos, la población es igual a la muestra". (Castro Márquez, 2003, p. 69).

\section{RESULTADOS}

\section{Encuesta a los agricultores de invernaderos solares del sector villa concepción de la ciudad de Potosí}

\section{Control de la humedad del aire}

Se determinó que no se realiza el control de la humedad del aire en los invernaderos solares de la zona villa concepción de la ciudad de Potosí, y ya que, el $86 \%$ de los agricultores no cuentan con herramientas ni conocimientos sobre este factor climático, el $14 \%$ lo hace de manera empírica, según sus conocimientos, abriendo las ventanas desde las 8:00 am hasta las 5:00 pm y de esa manera poder enfriar el lugar en tiempos de verano o calentar en épocas de invierno tal cual se muestra en la tabla 1. 
Tabla 1. Humedad del aire.

\begin{tabular}{ccc}
\hline ALTERNATIVAS & FRECUENCIA & $\mathbf{\%}$ \\
\hline $\mathrm{Si}$ & 2 & 14 \\
$\mathrm{No}$ & 12 & 86 \\
\hline Total & $\mathbf{1 4}$ & $\mathbf{1 0 0}$
\end{tabular}

\section{Control de la humedad del suelo y manera de realizar el control}

En la tabla 2, se muestra que un $71 \%$ de los encuestados realiza el control de la humedad del suelo pero de manera empírica, usando palitos, madera, u otro material para ver si esta húmeda la tierra y así poder verificar, pero el $29 \%$ de la población no realiza el control de este factor climático ya que lo ven un tanto innecesario.

Tabla 2. Humedad del suelo

\begin{tabular}{ccc}
\hline ALTERNATIVAS & FRECUENCIA & $\mathbf{\%}$ \\
\hline $\mathrm{Si}$ & 10 & 71 \\
$\mathrm{No}$ & 4 & 29 \\
\hline Total & $\mathbf{1 4}$ & $\mathbf{1 0 0}$ \\
\hline
\end{tabular}

\section{Control de la temperatura diurna}

En la tabla 3, se muestra que el $86 \%$ de la población controla la temperatura diurna, abren las ventanas una vez que salga el sol, para que pueda ventilar el lugar y baje la temperatura, en el caso de que se encuentre alta, mientras que el 14\% de la población no hace el control de la temperatura diurna, ya que, en horas de la mañana les quita tiempo y algunas veces olvidan abrir las ventanas.

Tabla 3. Temperatura diurna

\begin{tabular}{ccc}
\hline ALTERNATIVAS & FRECUENCIA & $\mathbf{\%}$ \\
\hline $\mathrm{Si}$ & 12 & 86 \\
$\mathrm{No}$ & 2 & 14 \\
\hline Total & $\mathbf{1 4}$ & $\mathbf{1 0 0}$
\end{tabular}

\section{Control de la temperatura nocturna}

Según se aprecia en la tabla 4 el $86 \%$ de los encuestados solo cierran las ventanas a partir de las 17:00 pm para mantener el calor generado por el sol, el 14\% no hace el control de la temperatura nocturna debido a la falta de conocimiento e instrumentos.

Tabla 4. Temperatura nocturna

\begin{tabular}{ccc}
\hline ALTERNATIVAS & FRECUENCIA & $\mathbf{\%}$ \\
\hline $\mathrm{Si}$ & 12 & 86 \\
$\mathrm{No}$ & 2 & 14 \\
\hline Total & $\mathbf{1 4}$ & $\mathbf{1 0 0}$
\end{tabular}




\section{Control del dióxido de carbono}

En la tabla 5, se muestra, que la totalidad de los encuestados no realizan el control del dióxido de carbono, debido a que desconocen la importancia que proporciona al cultivo y además que ayuda a la fotosíntesis.

Tabla 5. Dióxido de carbono

\begin{tabular}{ccc}
\hline ALTERNATIVAS & FRECUENCIA & $\mathbf{\%}$ \\
\hline $\mathrm{Si}$ & 0 & 0 \\
$\mathrm{No}$ & 14 & 100 \\
\hline Total & $\mathbf{1 4}$ & $\mathbf{1 0 0}$
\end{tabular}

Testimonios del dueño del invernadero la lechuguita de la ciudad de Potosí

\section{Dificultades presentes al momento de medir la humedad, temperatura $y$ dióxido de carbono en invernaderos solares}

El encargado del invernadero mencionó, que no tiene problemas en medir la temperatura, ya que cuenta con un termómetro, pero uno de los problemas es que sube y baja de manera constante, lo cual genera algunas plagas dentro del invernadero, de la humedad no cuenta con herramientas, lo cual es difícil saber cuándo sube o baja y del suelo es otro problema porque tiene que pinchar con un palito para saber si la tierra esta seca o no, de los demás factores no se tiene un control porque carece de conocimiento y herramientas.

\section{Herramientas o técnicas que se usa para medir la humedad relativa y del suelo}

El encargado del invernadero mencionó que de la humedad del aire (relativa) no se hace ningún control, porque no se cuenta con las herramientas necesarias, pero una de las técnicas es abrir todas las mañanas las ventanas para que se pueda ventilar el lugar y mantener más fresco el ambiente, de la humedad del suelo se revisa todos los días para verificar si la tierra está seca, se pincha con un palito y si este sale con algo de tierra entonces no necesita riego porque indica que aún está húmedo, caso contrario se debe regar.

\section{Herramientas o técnicas que se usa para medir la temperatura}

El encargado del invernadero indicó que para el control de la temperatura, se utiliza un termómetro, con la única finalidad de medir la temperatura del ambiente, y como técnica abren las ventanas cuando la temperatura excede del $35 \%$ caso contrario no se realiza ninguna acción, la supervisión lo realiza cada 2 veces al día.

\section{Herramientas o técnicas se usa para medir el nivel de dióxido de carbono}

Sobre el dióxido de carbono no tiene ningún conocimiento, por lo cual no se realiza ningún control sobre este factor.

\section{Factores climáticos son importantes}

Los factores más importantes son la temperatura y humedad, ya que depende de ellos si habrá buenas cosechas, de los demás factores no tiene conocimiento por lo cual no lo ve como primordial. 
Observación a todos los invernaderos del sector villa concepción

De la observación realizada a los invernaderos de la zona villa concepción, se pudo evidenciar que la mayoría de los invernaderos no realiza un control de la temperatura adecuada, tanto diurna como nocturna, los agricultores solo abren las ventanas durante todo el día siempre y cuando el clima está en condiciones favorables.

Para el control de la humedad del aire, solo abren las ventanas todas las mañanas, para que pueda ventilar el lugar. Pero según la observación que se realizó varia de un momento a otro; es decir baja y sube constantemente, lo cual provoca plagas en las plantas. De la observación realizada en los diferentes invernaderos para la humedad del suelo el $50 \%$ verifican con una varilla si la tierra esta húmeda para saber si necesitan riego o no, pero el otro $50 \%$ solo hacen la verificación del exterior de la tierra por observación.

Se pudo observar que no realizan ningún control del dióxido de carbono

Desarrollo de los componentes del sistema automatizado para regular el comportamiento de los factores climáticos en el cultivo de hortalizas en invernadero solar la lechuguita de la ciudad de Potosí.

Para la elaboración del sistema y tomando los factores de control del comportamiento de los cambios climáticos del cultivo de hortalizas en invernaderos solares, se utilizó la metodología XP ya que se adecua más al proyecto.

\section{Metodología XP}

Para Beck (1999), la programación extrema o eXtreme Programming (XP) es una metodología de desarrollo de la ingeniería de software, formulada por él.

Se puede considerar la programación extrema, como la adopción de las mejores metodologías de desarrollo de acuerdo a lo que se pretende llevar a cabo con el proyecto, y aplicarlo de manera dinámica durante el ciclo de vida del software.

\section{Diseño}

La metodología XP hace especial énfasis en los diseños simples y claros.

a) Simplicidad, un diseño simple se implementa más rápidamente que uno complejo. Por ello XP propone implementar el diseño más simple posible que funcione.

b) Metáfora para el sistema, XP sugiere utilizar este concepto como una manera sencilla de explicar el propósito del proyecto, así como guiar la estructura del mismo. Una buena metáfora debe ser fácil de comprender para el cliente y a su vez debe tender suficiente contenido como para que sirva de guía a la arquitectura del proyecto.

c) Riesgos, si surgen problemas potenciales durante el diseño, X.P. sugiere utilizar una pareja de desarrolladores para que investiguen y reduzcan al máximo el riesgo que supone ese problema.

d) Funcionalidad extra, nunca se debe añadir funcionalidad extra al programa aunque se piense que en un futuro será utilizada. Sólo el 10\% de la misma es utilizada, lo que implica que el desarrollo de funcionalidad extra es un desperdicio de tiempo y recursos. 
e) Refactorización, consiste en escribir nuevamente parte del código de un programa, sin cambiar su funcionalidad, a los efectos de crearlo más simple, conciso y estable. La metodología XP sugiere re codificar cada vez que sea necesario.

f) Tarjetas CRC, el uso de las tarjetas C.R.C. (Class, Responsabilities and Collaboration), permiten al programador centrarse y apreciar el desarrollo orientado a objetos olvidándose de los malos hábitos de la programación procedural clásica. Las tarjetas C.R.C. representan objetos; la clase a la que pertenece el objeto se puede escribir en la parte de arriba de la tarjeta, en una columna a la izquierda se pueden escribir las responsabilidades $\mathrm{u}$ objetivos que debe cumplir el objeto y a la derecha, son las clases que colaboran con cada responsabilidad.

\section{Prueba}

a) Pruebas unitarias, todos los módulos deben pasar las pruebas unitarias antes de ser liberadas o publicadas, y deben pasar correctamente las pruebas unitarias, es lo que habilita que funcione la propiedad colectiva del código.

b) Pruebas de aceptación, son creadas en base a las historias de usuarios, en cada ciclo de la iteración del desarrollo. El cliente debe especificar uno o diversos escenarios para comprobar que una historia de usuario ha sido correctamente implementada. Asimismo, en caso de que fallen varias pruebas, deben indicar el orden de prioridad de resolución. (Joskowicz, 2008)

\section{Costos de implementación del proyecto}

Tabla 6. Requerimientos de hardware

\begin{tabular}{|c|c|c|}
\hline HARDWARE & DESCRIPCIÓN & PRECIO \\
\hline Celular & Versión de Android 5.1 .1 & $500 \mathrm{Bs}$ \\
\hline Placa arduino & Mega 2560 & 90 Bs. \\
\hline Sensor de temperatura yhumedad & DHT11 & 15 Bs. \\
\hline $\begin{array}{c}\text { Sensor de humedad del } \\
\text { suelo }\end{array}$ & HL69 & $15 \mathrm{BS}$. \\
\hline $\begin{array}{l}\text { de dióxido de } \\
\text { carbono }\end{array}$ & MQ2 & $20 \mathrm{Bs}$. \\
\hline Mini bomba de agua & $120 \mathrm{~L} / \mathrm{H}$ & 25 Bs. \\
\hline Módulo bluetooth & HC-05 & $50 \mathrm{Bs}$. \\
\hline Módulo DS3231 & $12 \mathrm{C}$ & $30 \mathrm{Bs}$. \\
\hline Driver dual para motores & L298N & $50 \mathrm{Bs}$. \\
\hline Mini protoboard & & 15 Bs. \\
\hline Ventiladores & & 40 Bs. \\
\hline Cables jumper & & $50 \mathrm{Bs}$. \\
\hline
\end{tabular}


Tabla 7. Requerimientos de software

\begin{tabular}{lll}
\hline \multicolumn{1}{c}{ SOFTWARE } & \multicolumn{1}{c}{ DESCRIPCIÓN } & \multicolumn{1}{c}{ PRECIO } \\
\hline App Inventor & Para desarrollar la aplicación & Licencia libre \\
Arduino IDE & Para la programación de los sensores & Licencia libre \\
Fritzing & Para el diseño del circuito electrónico & Licencia libre \\
Magic Draw & Para el diseño de los diagramas del proyecto & Licencia flotante \\
\hline
\end{tabular}

Tabla 8. Cálculo del precio del sistema

\begin{tabular}{cllc}
\hline $\mathbf{N}^{\circ}$ & \multicolumn{1}{c}{ Nombre del sistema } & Características & Costo \$ \\
\hline $1=$ "P" & $\begin{array}{l}\text { Diseño de un sistema } \\
\text { automatizado a través de } \\
\text { interfaces remotas. }\end{array}$ & $\begin{array}{l}\text { Utilización de la tecnología Arduino } \\
\text { plataforma deprogramación de } \\
\text { códigoabierto. }\end{array}$ & $\mathbf{3 . 0 0 0}$ \\
$2=$ "M" & $\begin{array}{l}\text { Diseño e implementación de un } \\
\text { sistema automatizado, para } \\
\text { regular el comportamiento de los } \\
\text { factores climáticos. }\end{array}$ & $\begin{array}{l}\text { Un sistema que regule } \\
\text { automáticamente los } \\
\text { comportamientos de los factores } \\
\text { climáticos. }\end{array}$ & $\mathbf{1 7 . 0 0 0}$ \\
$3=$ "O" & $\begin{array}{l}\text { Diseño e implementación del } \\
\text { monitoreo y registro activación de } \\
\text { los sensores en tiempo real. }\end{array}$ & $\begin{array}{l}\text { Un sistema que muestre el monitoreo } \\
\text { y registro deactivación de los } \\
\text { sensoresen tiempo real mediante la } \\
\text { app vía bluetooth. }\end{array}$ & $\mathbf{5 . 0 0 0}$ \\
\hline
\end{tabular}




\section{Desarrollo de la propuesta}

\section{Proceso de negociación}

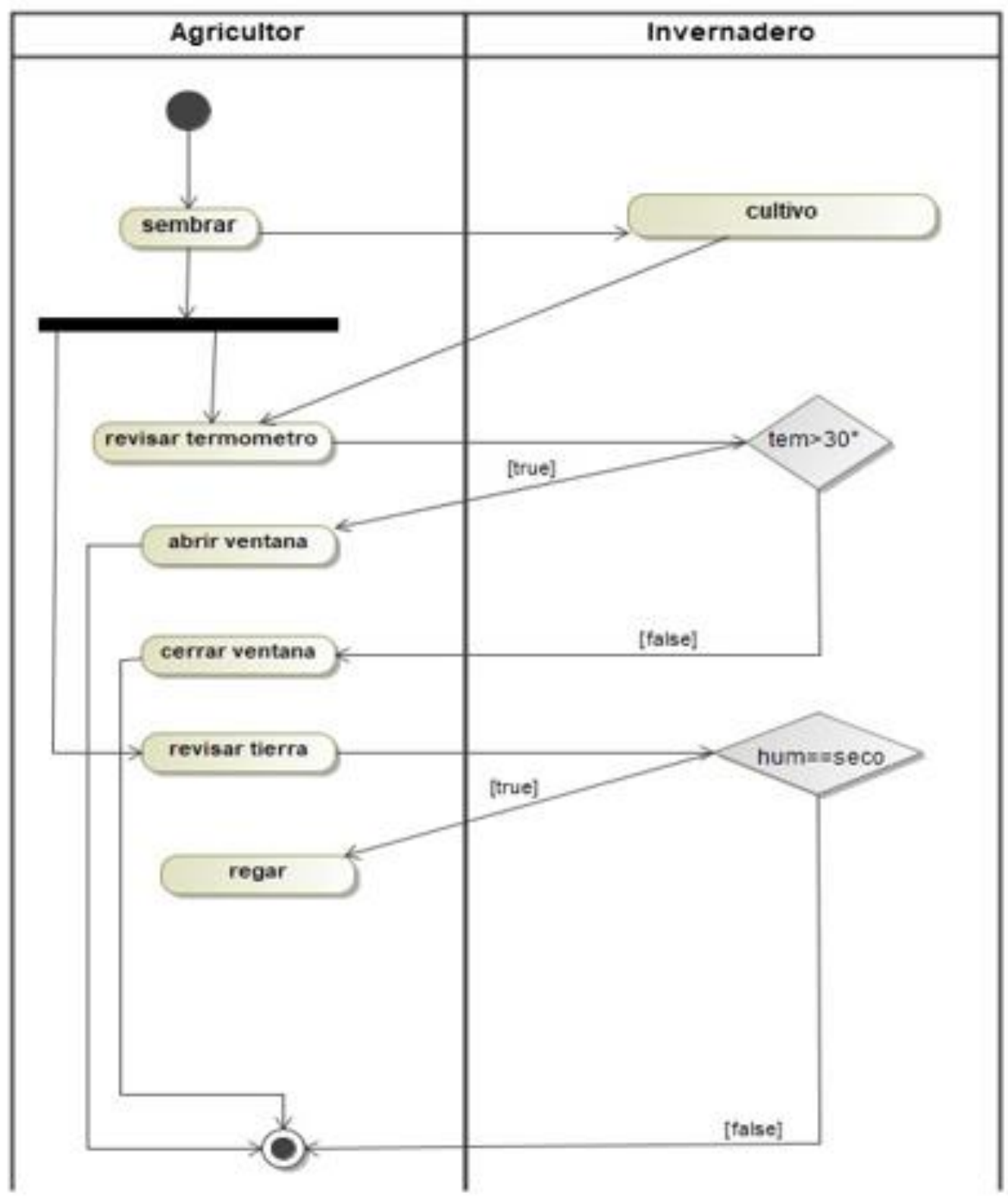

Figura 8. Diagrama de actividades de negocio 


\section{Tareas de ingeniería}

Tabla 9.Tarea de ingeniería: Gestión usuario: Diseño de la interfaz acceder al sistema.

\begin{tabular}{cc}
\hline \multicolumn{2}{c}{ Tareas de Ingeniería } \\
\hline Número de tarea: 1 & Número de historia: 1 \\
Nombre de la tarea: Diseño de la interfaz acceder al sistema & Puntos estimados: 0.5 \\
\hline Tipo de tarea: Desarrollo & Fecha final: 09/11/2018 \\
\hline Fecha inicio: 05/ 11/2018 & Programador responsable: Mónica Joselin Ala Delgado \\
\hline $\begin{array}{l}\text { Descripción: Se muestra la interfaz para acceder al sistema, en el cual los usuarios } \\
\text { podrán ingresar su nombre de usuario y contraseña. Además mostrará los datos del } \\
\text { ambiente del invernadero en tiempo real. }\end{array}$ \\
\hline
\end{tabular}

Tabla 10. Tarea de ingeniería 2: Gestión usuario: Diseño de la interfaz gestión usuario

\begin{tabular}{cc}
\hline \multicolumn{2}{c}{ Tareas de Ingeniería } \\
\hline Numero de tarea: 2 & Numero de historia: 1 \\
\hline Nombre de la tarea: Diseño de interfaz gestión usuario. & Puntos estimados: 0.5 \\
\hline Tipo de tarea: Desarrollo & Fecha final: 14/11/2018 \\
\hline Fecha inicio:12/11/2018 & Programador responsable: Mónica Joselin Ala Delgado \\
\hline $\begin{array}{l}\text { Descripción: Se muestra el diseño de la interfaz de la gestión usuario, en el cual se } \\
\text { manipula varias acciones, como agregar y editar los datos: nombre, apellidos } \\
\text { y usuario. }\end{array}$ \\
\hline
\end{tabular}

Tabla 11. Tarea de ingeniería 3: Gestión usuarios: Nuevo/editar

\begin{tabular}{cc}
\hline \multicolumn{2}{c}{ Tareas de Ingeniería } \\
\hline \multicolumn{1}{c}{ Nombre de la tarea: Nuevo editar } \\
\hline Numero de tarea: 3 & Puntos estimados: 0.5 \\
\hline Tipo de tarea: Desarrollo & Fecha final: 20/11/2018 \\
\hline Fecha inicio:15/11/2018 & Programador responsable: Mónica Joselin Ala Delgado \\
\hline $\begin{array}{l}\text { Descripción: Se observa la lista de los usuarios de los cuales se pueden modificar el } \\
\text { nombre, apellidos y nombre de usuario. Si se selecciona nuevo aparece la ventana } \\
\text { deregistro. }\end{array}$ \\
\hline
\end{tabular}


Tabla 12. Tarea de ingeniería 4: Gestión taxonomía: Diseño de la interfaz de gestión taxonomía.

\section{Tareas de Ingeniería}

Numero de tarea: $4 \quad$ Numero de historia: 2

Nombre de la tarea: Diseño de la interfaz de gestión taxonomía

Tipo de tarea: Desarrollo Puntos estimados: 0.5

Fecha inicio: 21/11/2018 Fecha final: 22/11/2018

Programador responsable: Mónica Joselin Ala Delgado

Descripción: Se muestra el diseño de la interfaz de gestión taxonomía.

Tabla 13. Tarea de ingeniería 5: Gestión taxonomía: Nuevo/editar/eliminar taxonomía

\begin{tabular}{cc}
\hline \multicolumn{2}{c}{ Tareas de Ingeniería } \\
\hline Número de tarea: 5 & Número de historia: 2 \\
\hline Nombre de la tarea: Nuevo editar eliminar taxonomía & Puntos estimados: 0.5 \\
\hline Tipo de tarea: Desarrollo & Fecha final: $28 / 11 / 2018$ \\
\hline Fecha inicio: $23 / 11 / 2018$ & Programador responsable: Mónica Joselin Ala Delgado \\
\hline $\begin{array}{l}\text { Descripción: Se observa la lista de las hortalizas de los cuales se pueden modificar la } \\
\text { familia, nombre común o científico, además también se puede eliminar, si se } \\
\text { selecciona nuevo aparece la ventana para registrar. }\end{array}$ \\
\hline
\end{tabular}


Diseño. Proceso para el registro de datos de la taxonomía en el sistema

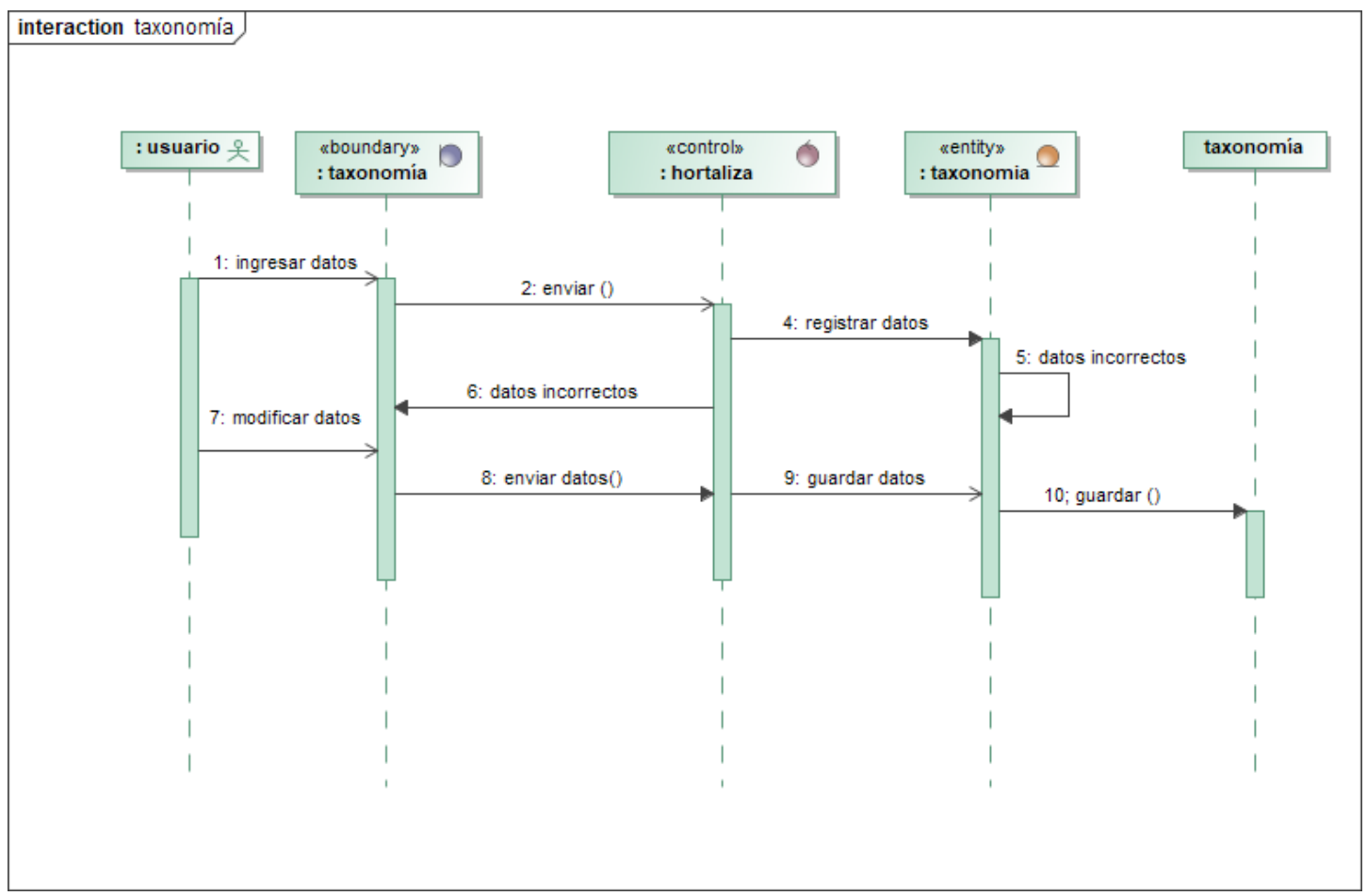

Figura 9. Diagrama de secuencia: Taxonomía

\section{Desarrollo para guardar los datos de la taxonomía}

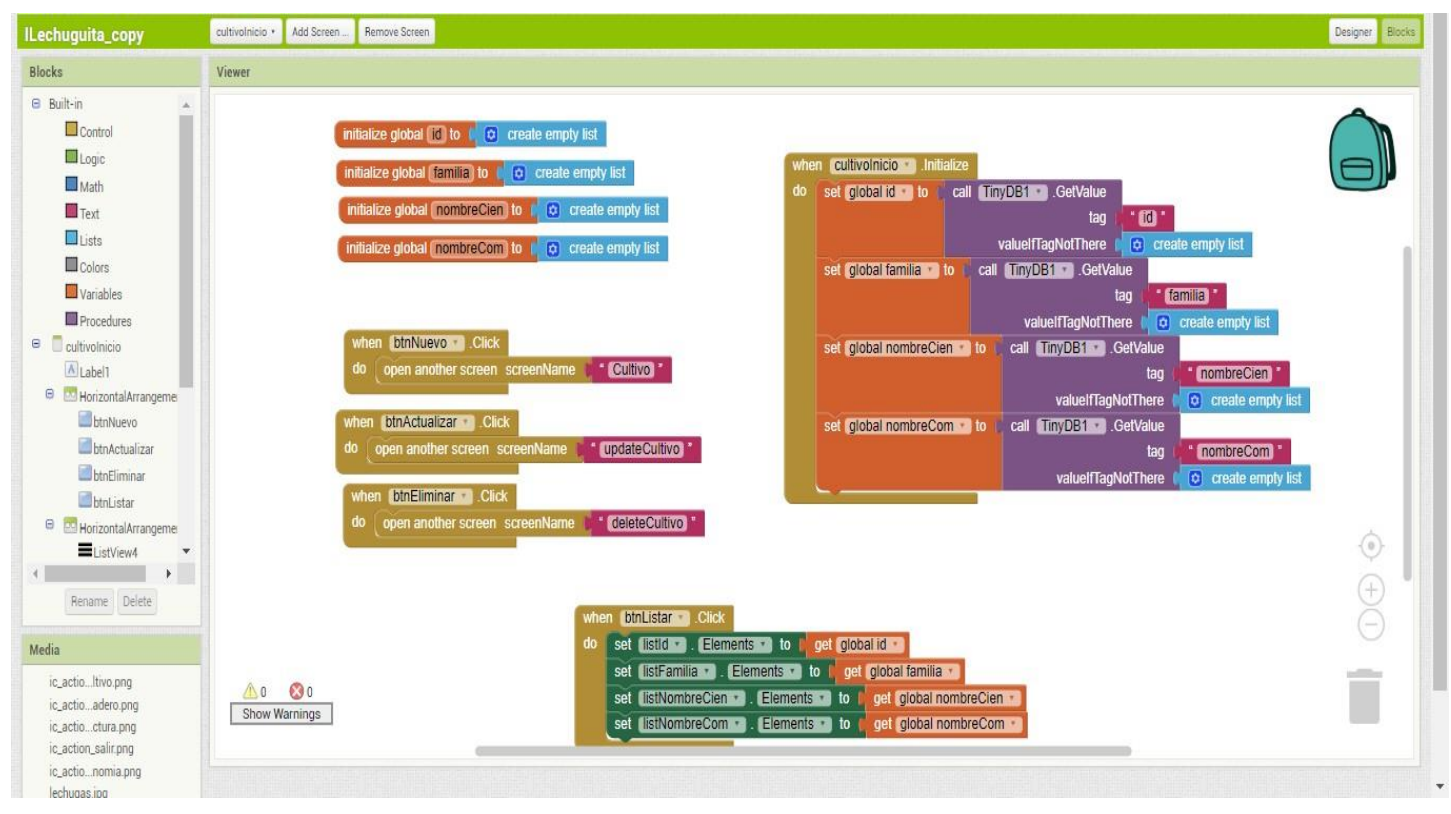

Figura 10. Guardar los datos de la taxonomía 


\section{Caso de prueba: Gestión taxonomía}

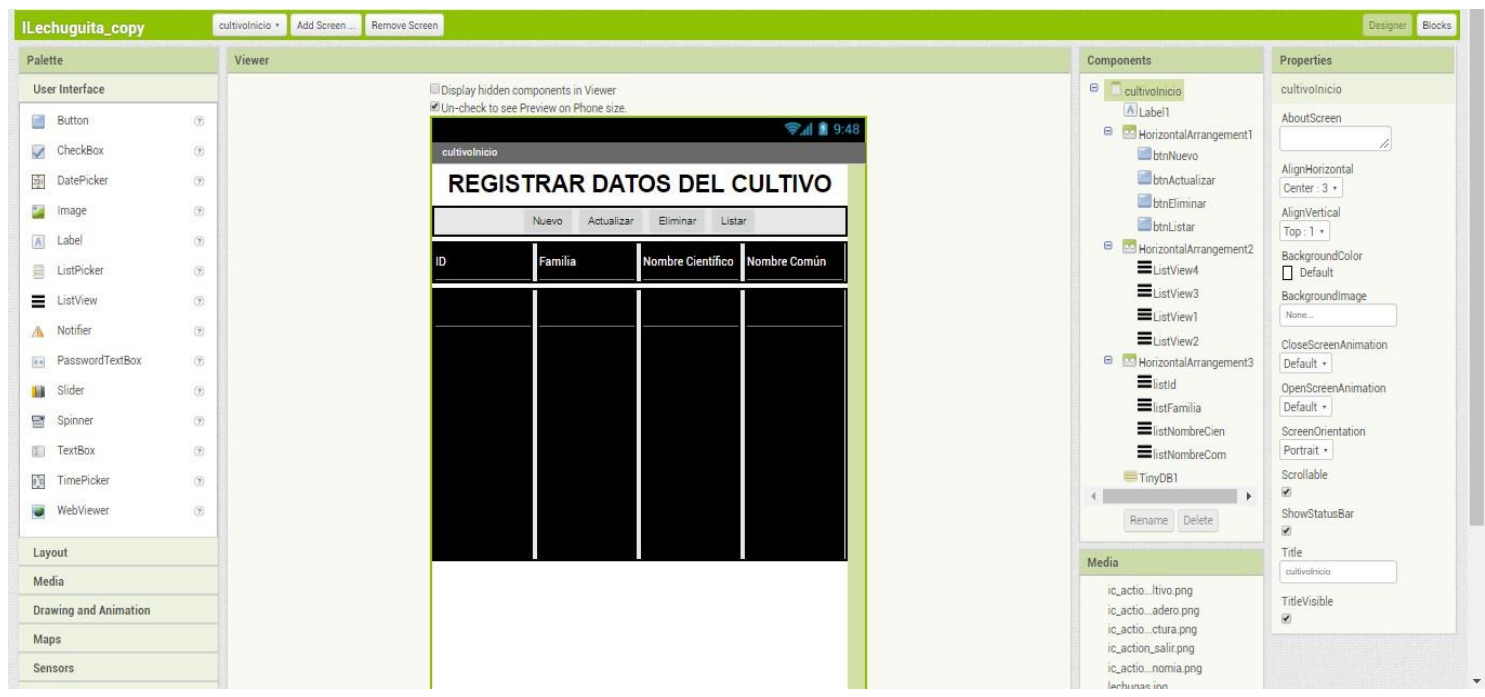

Figura 11. Gestión taxonomía

\section{CONCLUSIONES}

Con la sistematización de los fundamentos teóricos de los factores climáticos en invernaderos solares basándose en antecedentes históricos, en definiciones de los distintos autores, se fue profundizando en el comportamiento de los factores climáticos, se ha logrado determinar y estudiar todos los elementos que sustentan la investigación, esto se fue integrando con atribución al marco teórico a través del análisis.

El diagnóstico respecto a la inspección de los invernaderos solares de la ciudad de Potosí, se realizó sobre el estado actual en la que se encuentran el control de los factores climáticos, con los instrumentos de recolección de datos se evidenció que no se realizan ningún control de la taxonomía de los cultivos. Se comprobó que los agricultores necesitan un sistema automatizado para controlar todos los factores y realizar un monitoreo en tiempo real.

Se cumplió con la propuesta de desarrollo de los componentes del sistema automatizado para regular el comportamiento de los factores climáticos en el cultivo de hortalizas en el invernadero solar la Lechuguita de la ciudad de Potosí, donde se utilizó las herramientas que conforman el sistema, optando utilizar App Inventor 2, Arduino, la metodología XP y finalmente culminando con las pruebas correspondientes.

\section{REFERENCIAS}

Alvarado V., y Urrutia, G. (2003). Invernaderos. Agroeconómico de la fundación Chile, 11

Beck, K. (1999). Extreme Programming Explained. Embrace Change. Pearson Education

Castro Márquez, F. (2003). El proyecto de investigación y su esquema de elaboración. Caracas: Uyapar

Estrada, J. (2012). Guía para la construcción de invernaderos o fitotoldos. Obtenido de FAO Bolivia: http://www.fao.org/3/a-as968s.pdf

FAO. (2004). Buenas prácticas agrícolas BPA- en la protección de tomate bajo condiciones protegidas. $50-65$ 
InfoAgro. (s.f.).Obtenido de Principales tipos de invernaderos: http://canales.hoy.es/canalagro/datos/ industria_auxiliar/tipo_invernaderos2.h tm

Joskowicz, J. (2008). Reglas y Prácticas en eXtreme Programming. Manitoba

Juárez, P.; Bugarín, M.; Castro, R.; Sánchez,
A.; Cruz, E.; Juáres, C., y. Balois, R. (2011). Estructuras utilizadas en la agricultura protegida. fuente, 5-7

Scuitto, E., y Mascareña, J. (2000). Sistema de control automático de temperatura y ventilación de un Invernadero. Proyecto calafate, 6-8 